IMAGE ARTICLE

\title{
Interval Form of Carbon Monoxide Intoxication
}

\section{Ee Wei Lim*, Yee Hau Pang and Pavanni Ratnagopal}

National Neuroscience Institute, Singapore General Hospital, Singapore

*Corresponding author: Ee Wei Lim, MD, National Neuroscience Institute, Singapore General Hospital, Campus 20 College Road, Academia, Singapore,E-mail: lim.ee.wei@singhealth.com.sg

A 41-year-old gentleman attempted suicide by burning charcoal in an enclosed room. He was found unconscious and was intubated for poor Glasgow Coma Scale (GCS). He was successfully extubated and transferred to our hospital on day 6. Initial physical examination showed no neurological deficits with normal scoring of Mini-Mental State Examination (MMSE) and Montreal Cognitive Assessment (MOCA). Computerized Tomography (CT scan) of the brain was done on day 8 (Figure 1). Magnetic Resonance Imaging (MRI) of brain done on day 10 was shown in Figure 2A and Figure 2B. He had major depression and was transferred to the care of psychiatrist. Initial neuropsychological assessment showed subtle deficits in learning, retrieval of information and verbal fluency.

About 2 weeks later (around 3 weeks from the incidence), he was noticed to be less communicative and having difficulties in mobilization and performing activities of daily living. He was then transferred back for further evaluation. He was noticed to be withdrawn and not communicative. He had magnetic gait (hardly walked with freezing), limbs and truncal rigidity. Repeat MRI brain was done (Figure 3A, Figure 3B, Figure 3C, Figure 3D). He was treated with madopar and fluoxetine. He subsequently underwent rehabilitation.

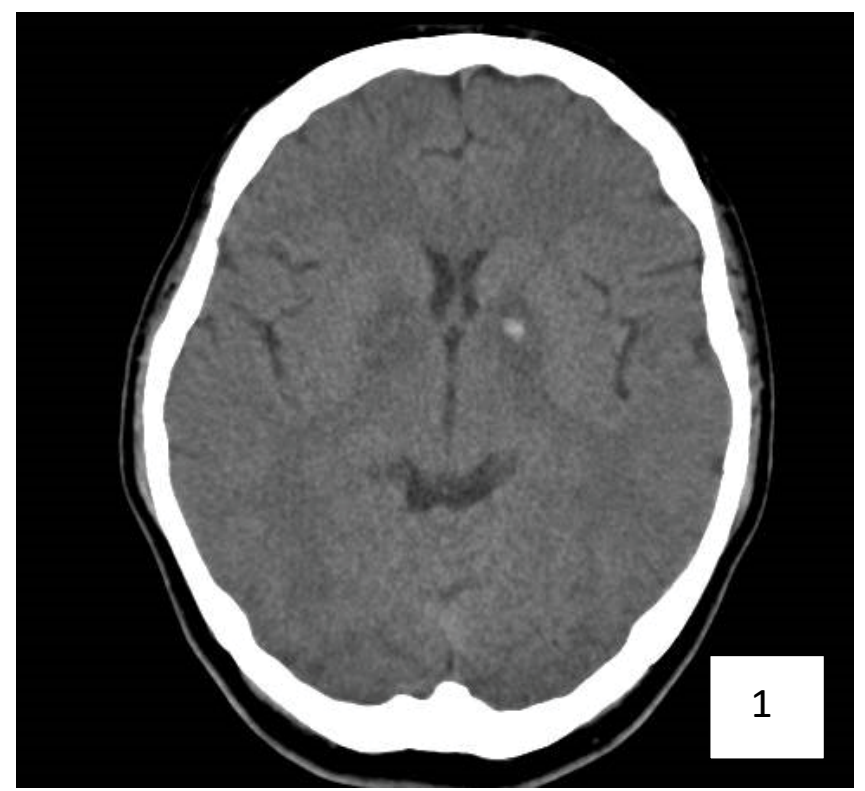

Figure 1: CT brain on day 8: symmetrical hypodensity at the globus pallidus with a small focus of acute hemorrhage on the left.

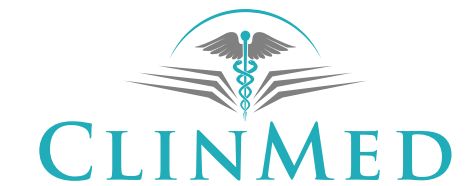

INTERNATIONAL LIBRARY
Citation: Lim EW, Pang YH, Ratnagopal P (2017) Interval Form of Carbon Monoxide Intoxication. Clin Med Img Lib 3:062. doi.org/10.23937/2474-3682/1510062

Received: November 29, 2016; Accepted: April 19, 2017; Published: April 22, 2017

Copyright: (C) 2017 Lim EW, et al. This is an open-access article distributed under the terms of the Creative Commons Attribution License, which permits unrestricted use, distribution, and reproduction in any medium, provided the original author and source are credited. 

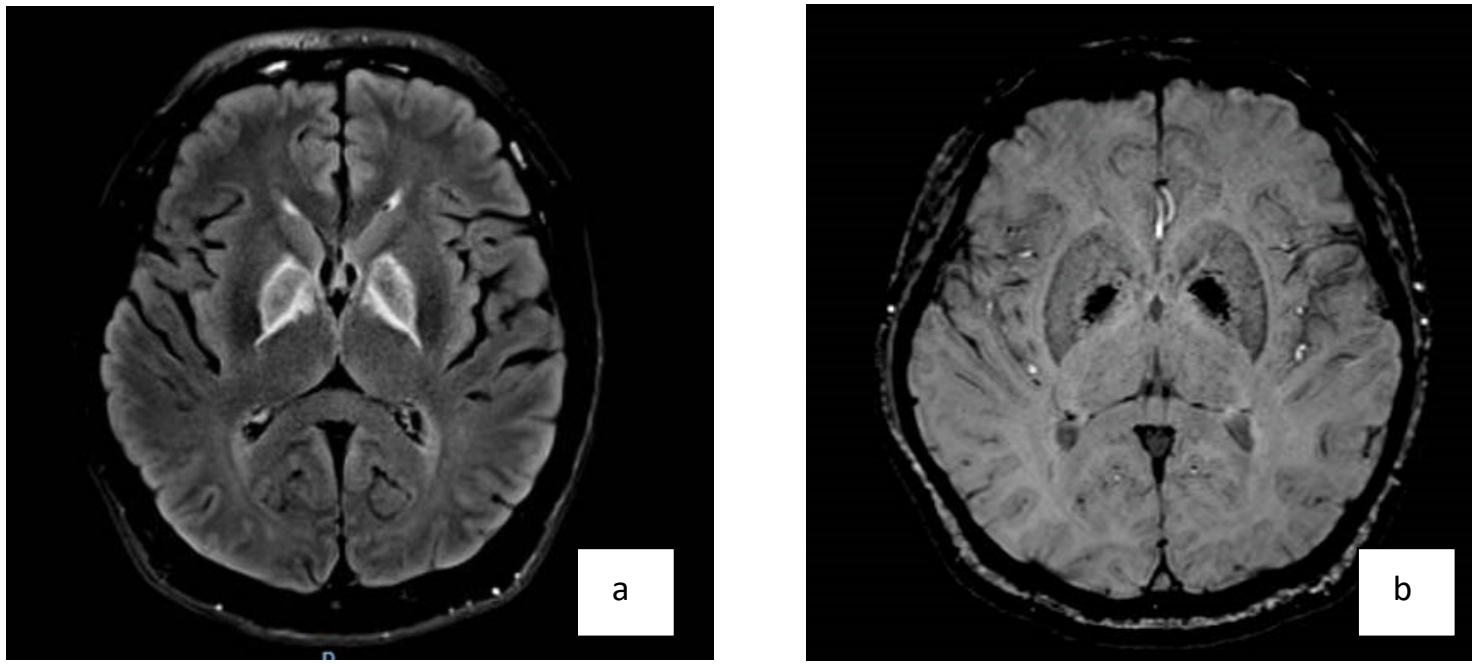

Figure 2: MRI brain done on day 10 .

A,B) High FLAIR signal with hyperintense rim in the bilateral globus pallidus with susceptibility.
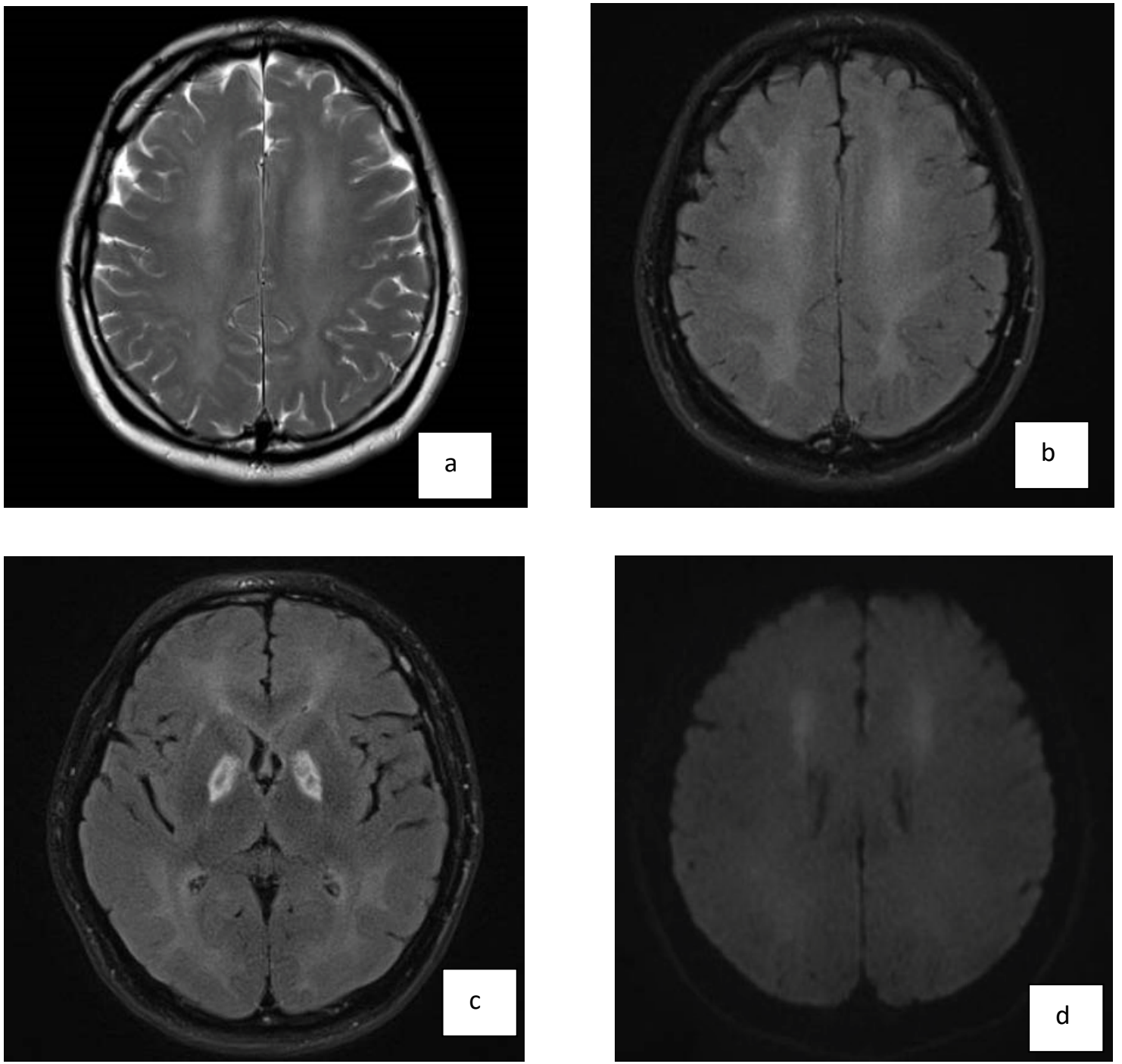

Figure 3: MRI brain done at day 31.

A,B) Interval progression of extensive white matter changes with near confluent T2 weighted and FLAIR hyperintensity in both cerebral hemispheric white matter and near total loss of grey-white matter differentiation, in keeping with delayed demyelination.

Figure 3C: Interval decrease in swelling with residual cystic encephalomalacia in both globus pallidus.

Figure 3D: Interval areas of restricted diffusion in both superior frontal subcortical white matter/anterior corona radiata indicating ongoing neuronal white matter injury. 
6 months after the incidence, he improved significantly with residual mildly increased tone over lower limbs. He was independent in all activities of daily living. MMSE scoring was 28. Neuropsychological assessment done one year later showed slowed processing speed, impaired visuo-spatial construction, reduced mental flexibility and attention. Diagnosis of mild cognitive impairment was made.

Our case illustrates a case of Carbon Monoxide (CO) poisoning with both early and late complications. Our patient had poor mental state immediately after poisoning leading to intubation. The lucid interval, which describes interval between acute phase of intoxication and emergence of the delayed neurological sequelae, be it mental deterioration (cognitive impairment), parkinsonism, gait disturbances and mutism in our case. He had made a substantial recovery and complicated by mild cognitive impairment.

There are two major pathophysiologic mechanism of $\mathrm{CO}$ toxicity, which are hypoxic and cellular theories. $\mathrm{CO}$ strongly binds to hemoglobin forming carboxy-hemoglobin in the blood stream and this can cause profound hypoxic injury. $\mathrm{CO}$ inhibits the mitochondrial electron transport enzyme system and causes brain lipid peroxidation, leading to the interval form of $\mathrm{CO}$ toxicity [1].

Globus pallidus is the most commonly involved site in $\mathrm{CO}$ poisoning [1]. The predilection for the globus pallidus is unclear. However, it is probably related to $\mathrm{CO}$ binding to iron-rich globus pallidus or hypotensive effects of $\mathrm{CO}$ toxicity in the watershed arterial territory. Diffuse cerebral white matter demyelination (Figure 3A, Figure $3 \mathrm{~B}$ ) like what was observed in our case is believed to be responsible for interval form of $\mathrm{CO}$ poisoning. Restricted diffusion in both superior frontal subcortical white matter (Figure 3D) have shown the development of delayed cytotoxic edema, possibly due to delayed cell death and demyelination.

\section{Reference}

1. Lo CP, Chen SY, Lee KW, Hsueh CJ, Huang GS, et al. (2007) Brain injury after acute carbon monoxide poisoning: early and late complications. AJR Am J Roentgenol 189: 205-211. 